\title{
Filigrane
}

Écoutes psychothérapiques

\section{Ce corps qui parle pour moi}

\section{Diane Aubin}

Volume 18, numéro 2, automne 2009

Le corps. Sur le divan. Dans le fauteuil II

URI : https://id.erudit.org/iderudit/039286ar

DOI : https://doi.org/10.7202/039286ar

Aller au sommaire du numéro

Éditeur(s)

Revue Santé mentale au Québec

ISSN

1192-1412 (imprimé)

1911-4656 (numérique)

Découvrir la revue

\section{Citer cet article}

Aubin, D. (2009). Ce corps qui parle pour moi. Filigrane, 18(2), 16-30. https://doi.org/10.7202/039286ar

\section{Résumé de l'article}

La prise en compte du langage corporel, du non verbal et des réactions contre-transférentielles dans l'intervention et plus particulièrement en psychothérapie est une démarche reconnue quoique d'importance inégale selon les approches ou points de vue. En ce qui concerne l'abord des jeunes de la rue ou en grande difficulté, la lecture et l'écoute du langage du corps deviennent essentielles voire incontournables. En effet, celui-ci participe à l'articulation des formes de détresse et d'affirmation de ces jeunes, lesquelles peuvent susciter des réactions très intenses chez l'intervenant qui a comme projet d'aller à leur rencontre. Le travail en équipe et l'écoute de son propre corps deviennent pour l'intervenant des impératifs tout aussi incontournables afin de préserver sa propre vitalité et son intégrité psychique. Interroger le corps, le sien et celui de l'autre est donc au coeur de la clinique de la précarité et de l'addiction.
Ce document est protégé par la loi sur le droit d'auteur. L'utilisation des services d’Érudit (y compris la reproduction) est assujettie à sa politique d'utilisation que vous pouvez consulter en ligne.

https://apropos.erudit.org/fr/usagers/politique-dutilisation/ 


\section{Ce corps qui parle pour moi}

\section{diane aubin}

La prise en compte du langage corporel, du non verbal et des réactions contretransférentielles dans l'intervention et plus particulièrement en psychothérapie est une démarche reconnue quoique d'importance inégale selon les approches ou points de vue. En ce qui concerne l'abord des jeunes de la rue ou en grande difficulté, la lecture et l'écoute du langage du corps deviennent essentielles voire incontournables. En effet, celui-ci participe à l'articulation des formes de détresse et d'affirmation de ces jeunes, lesquelles peuvent susciter des réactions très intenses chez l'intervenant qui a comme projet d'aller à leur rencontre. Le travail en équipe et l'écoute de son propre corps deviennent pour l'intervenant des impératifs tout aussi incontournables afin de préserver sa propre vitalité et son intégrité psychique. Interroger le corps, le sien et celui de l'autre est donc au cœur de la clinique de la précarité et de l'addiction.

Heeding body language and interpreting the impact of non verbal and countertransference responses in the course of intervention, and more specifically in the psychotherapeutic process, is a practice of varying importance in approaches adopted by caretakers and clinicians of diverse horizons and disciplines. When it comes to street youths or young people with severe difficulties, the way one listens to and reads body language becomes an essential and truly fundamental dimension of one's action and understanding. Indeed, body language concurs to the expression and articulation of various forms of distress and self affirmation in youth, and these patients often stir up intense reactions in clinicians involved with them. Consequently, close teamwork and attention to body reactions are considered as essential means to keep one's own vitality and psychic integrity. Questioning and interpreting body language, one's own and the other's, should be at the core of clinical intervention when confronted with youths living in precarious situations or afflicted with addiction.

Le corps occupe ainsi une place symbolique essentielle, et parfois bien malgré lui, car il marque le rapport qu'entretient l'individu avec le monde (Lambrette, 2008, 13)

\section{Le corps, cet Idéal du Moi ?}

$\mathrm{F}$ orce est de constater que nous vivons à une époque où nous sommes souvent tenus responsables du corps que l'on a puisque divers moyens, outils et technologies sont mis à notre disposition pour pouvoir le maîtriser et remédier aux divers malaises et imperfections qui le façonnent au gré de l'existence. Les jeunes aussi seraient interpellés par les impératifs et les dictats de la société de consommation dans laquelle ils grandissent, soumis aux messages de «valorisation des idéaux du corps », comme le souligne Birraux (2004, 93). De son côté, Le Breton rappelle justement que «le narcissisme d'aujourd'hui [...] est aussi le fait d'un travail sur soi» $(1990,173)$. Même les plus rebelles sont conviés à s'occuper de leur corps pour ne pas dire l'occuper, faute de repères ou de pouvoir investir d'autres territoires (Aubin, 2000)! 
L'une des caractéristiques de notre société contemporaine est la tendance à nous rendre responsable de notre propre corps et, ultimement, responsable de notre propre malheur ou de n'avoir pas su remédier à la situation qui nous afflige. Une époque où l'on est convié fortement à la recherche du bonheur, à construire le sien faute de le trouver (Bruckner, 2000). Nous nous voyons offrir mille et une façons de se construire une image, de tricoter son apparence, de façonner son bonheur et de soulager ses souffrances. Les drogues ayant perdu leur caractère sacré, elles ont acquis une valeur utilitaire et expérimentale qui autorise l'accès à des formes de régulation autonome des humeurs et des émotions. Dans certaines situations, elles peuvent même suppléer aux relations insatisfaisantes; à l'instar de nombreux adultes, les jeunes y trouvent, parmi d'autres formes de modalités relationnelles addictives, des amis fidèles, ersatz de relation qui peuvent les satisfaire à plus ou moins long terme. Devant tant d'emphase accordée au corps, à la recherche du plaisir et du bonheur, faut-il vraiment s'étonner des difficultés éprouvées par plusieurs jeunes à exprimer leurs émotions ou appréhender leur souffrance par le biais de la parole?

Les ouvrages qui traitent de l'investissement du corps et de ses attributs, avec l'engagement plus ou moins accentué dans des prises de risques, se sont multipliés au cours des dernières années (Birraux, 2004; Hachet, 2001, 2005; Le Breton, 2002, 2007 ; Pommereau, 2006). Afin de mieux comprendre les formes d'anxiété et de dépression qui participent des maux de notre siècle, philosophes et sociologues ont remis en question, outre «l'exigence de bonheur» (Bruckner, 2000), la pression exercée sur le moral des individus par les valeurs de performance et de rendement, cette «exigence d'autonomie» (Otero, 2005) qui favorise peut-être les conditions pour l'augmentation de l'anxiété et d'une forme de dépression dite narcissique, laquelle creuse son terreau dans la honte et le sentiment de faillite. Les lectures psychologique, psychiatrique et psychanalytique quant à elles rendent compte de l'émergence ou de l'augmentation des troubles relationnels, des organisations limites de la personnalité, des failles du narcissisme, des difficultés d'attachement, des distorsions du Moi ou des problèmes d'identité (Birraux, 2004 ; Cyrulnik, 2001; Jeammet et Corcos, 2001; Fœlsch, P. A., Odom, A. E. et Kernberg, O. F., 2008 ; Jacobson, 1954).

Certes, dans notre univers de plus en plus complexe, les réponses ne sont certainement pas simples et univoques et la réflexion demeure essentielle et passionnante. En ce qui me concerne, ma pratique clinique auprès de jeunes en très grande difficulté s'est construite et enrichie en puisant dans les perspectives sociale, anthropologique, culturelle, philosophique, pour ne nommer que celles-là. Il m'apparaît essentiel d'explorer et d'intégrer des points de vue provenant de divers champs d'études si l'on souhaite appréhender la complexité de l'homme moderne et son entrée dans son parcours d'adulte. J'invite le lecteur à explorer les questions fondamentales qui accompagnent les formes d'affirmation et d'expression de la souffrance des jeunes qui grandissent dans nos sociétés en mouvance. 


\section{Une clinique de la précarité et de l'addiction}

Cette entrée en matière me permet d'aborder un thème central dans la clinique de la précarité (Furtos, 1994; Ratier, 2007) et de l'addiction (McDougall, 1989; Pirlot, 2002) que représente essentiellement ma pratique dans un centre de jour ${ }^{1}$ qui offre des services à des jeunes en grande difficulté. Des jeunes qui ne s'inscrivent pas dans un parcours normatif et qui risquent de faire les frais d'exigences qui dépassent leurs capacités adaptatives. Des jeunes qui ont été ou sont forcément confrontés au manque et à la faille, et à leurs corollaires que sont les sentiments de vide, d'impuissance et de faillite. Je suis amenée à m'intéresser aux modalités ou stratégies de sauvegarde personnelle qu'ils ont mises en place pour la préservation de leur équilibre narcissique mis à mal.

Est-il besoin de rappeler qu'un bon nombre des jeunes marginaux ou décrocheurs ont fui ou tenté de composer avec des conditions de vie qui menaçaient leur intégrité physique ou psychologique. D'autres se sont retrouvés dans des impasses, soumis à des exigences qui dépassaient leurs capacités d'adaptation à un point donné. Leur condition actuelle constitue donc l'aboutissement d'expériences diverses, tantôt traumatiques, tantôt énigmatiques, et leur parcours jonché de défis et d'embûches est souvent à risque de déboucher sur une errance sans fin si les conditions ne sont pas mises en place pour stimuler leur résilience et relancer leur développement.

Par ailleurs, la prise en compte du langage corporel ou des messages véhiculés en dehors de la parole, ou sans que celle-ci ait priorité n'est pas un fait nouveau. Elle est même au cœur des missions humanitaires qui sont confrontées à des formes de silence, de non dit, de secret, de retrait, de méfiance, de sidération et de peur chez les populations vers lesquelles tendent leurs efforts. Chez les réfugiés et les exclus, les besoins de base ont été ou demeurent insatisfaits, frustrés, au pire niés ou bafoués. Celui ou celle qui veut comprendre et aider aura donc à déchiffrer un certain nombre de choses qui ne seront pas dites ni explicites. Les jeunes que je suis amenée à rencontrer ont donc été, pour la plupart, soumis à des secousses qui ont ébranlé leurs assises narcissiques ou l'évolution de leur construction identitaire. La prise en compte du langage du corps dans l'approche, l'abord ou l'accompagnement de ces jeunes sera donc au cœur d'un apprivoisement mutuel qui va se tisser au gré des allers et retours et des mouvements de rapprochement et de mise à distance qui forment mes interactions avec eux (Aubin, 2002). Faute de mots pour le dire ou se raconter, ils me donneront à percevoir l'indicible, entrevoir l'innommable ou ressentir l'impensable, avant de pouvoir avoir accès à leurs propres affects ou tolérer certaines des émotions que je devrai porter, contenir et restituer, le temps de reconstituer leur propre espace psychique et de réparer leur Moi-peau pour utiliser l'expression de Didier Anzieu (1994); et cela sera possible dans la mesure ou leurs mises en acte ne sont pas venues à bout de leurs ressources vitales et épuisé celles des ressources qui les accueillent ! Car, comme l'a souligné David Le Breton (1990), «la lutte chaque jour renouvelée pour la survie implique d'abord une lutte contre son propre corps ». 


\section{Des lieux de rencontres et d'échanges}

Une rencontre a toujours lieu quelque part. Depuis 1997, le centre de jour où je travaille accueille entre 150 et 200 jeunes âgés de 16 à 26 ans qui se marginalisent de par toutes sortes de conditions, avec ou contre leur gré. Leur premier objectif : manger, boire ou se réchauffer, ou encore, se déposer dans un lieu sûr, le temps de reprendre leur souffle, d'entrer en contact de manière sécuritaire avec quelqu'un, ou tout simplement être là, sans attente ou sans désir manifeste. Le degré « 0 » de l'objectif, pourrait-on dire, mais un objectif tout de même, un espace (ouvert, fermé, ambivalent) pour l'émergence d'un désir! C'est ainsi qu'au delà d'une demande d'aide, ou sous-jacente à celle-ci, peut se profiler une attente, s'esquisser un désir, lesquels auront besoin d'être reçus, traduits, formulés ou interprétés par l'autre pour pouvoir être conscients ou reconnus; l'adversité à laquelle sont confrontés nombre des jeunes n'autorisant pas toujours l'écoute de ses propres désirs puisque les exigences reliées à la précarité forcent l'attention sur les stratégies de survie (Flamand et Aubin, 2004).

Pour aller à leur rencontre, je bénéficie donc d'une structure d'accueil adaptée et de l'étayage relationnel que permettent le travail d'équipe et l'instauration de partenariats. L'hospitalité offerte à ces jeunes tient compte d'une phase d'apprivoisement mutuel; elle propose des avenues pour l'exploration de l'altérité (Gotman, 2001) et d'une nouvelle qualité de rapport à soi et à l'autre (Aubin, 2000, 2008). Comme ils ne sont pas forcés de formuler une demande d'aide pour avoir accès à nos services — celle-ci ne sera d'ailleurs peut-être jamais portée ou exprimée par eux-mêmes, nos premiers contacts et nos échanges peuvent prendre diverses formes, allant de salutations dans la cafétéria du centre de jour à une rencontre informelle dans un corridor ou la salle d'accueil, ou encore, à une rencontre formelle dans l'espace de mon bureau.

C'est toujours avec une certaine appréhension et de l'étonnement que je vais à la rencontre de jeunes qui se sont assis ou couchés par terre, ou qui se sont effondrés sur un banc ou sur une table de la cafétéria. J'ai encore l'habitude d'aller vérifier s'ils respirent encore, toujours étonnée et souvent attristée de l'apparente absence de souffrance ou d'émotion sur leur visage. Cet élan de ma part est une sorte de mouvement de reconnaissance, un prétexte pour leur signifier que j'accuse réception de leur présence, pour leur démontrer mon intérêt à les connaître, pour vérifier s'ils sont en lien avec un intervenant, ou encore leur signifier que leur condition m'interpelle, lorsque leur regard croise le mien ou que je cherche le leur. Même après toutes ces années, je ne m'habitue pas à la fatigue et l'abandon qui marque leur corps si jeune, même si leur visage reste figé, comme soucieux de rester inexpressif ou de ne pas ressentir la détresse qui marque pourtant leurs traits. Geste conscient de leur part? Plusieurs sont sans doute déjà happés par cette qualité particulière de souffrance que Furtos $(1994,17)$ appelle «une souffrance qui commence d'empêcher de vivre», ou encore celle «qui empêche de souffrir, donc de vivre». Affliction, désolation, agacement, accablement même, sont parmi les sensations et sentiments que j'éprouve à ces moments là ; et je suis même assez 
souvent envahie par l'indignation ou la tristesse qui me rappellent qu'il reste tant à faire et à bousculer pour que les choses changent...

Pourtant, j'aurai tout de même pris un peu de temps à demander à une jeune femme ou un jeune homme venu me rencontrer dans mon bureau s'il ou elle avait mangé ce matin, et s'il ou elle avait dormi la nuit dernière, et si oui, dans quel lieu. J'aurai aussi mis un peu de temps à réaliser que même si l'un de ces jeunes avait trouvé refuge dans un abri quelconque, il se pouvait qu'il n'ait pas réussi à s'endormir, pris par la peur qu'il ne lui arrive quelque chose, par un sentiment d'étrangeté ou la reviviscence de souvenirs traumatiques. C'est qu'ils n'en parlent pas d'emblée, et l'attitude de plusieurs laisse croire que le quotidien auquel ils sont confrontés n'est pas si pire que ça. Débrouillardise et conditions précaires oblige, plusieurs ont trouvé des moyens de s'affranchir de certains besoins, de ne pas ressentir la faim et le sommeil, au risque de perdre «le réflexe de manger» (l'expression est d'un jeune homme), la sensation de faim et le sentiment de peur. Car l'errance et la précarité peuvent conduire à une forme d'adaptation tout à fait particulière, paradoxale qui s'accompagne d'une transformation du ressenti en son absence (Furtos, 1994). De mon côté, surtout dans les premiers temps et équipée de mes repères normatifs, il est certain que je devais me préserver de découvrir derrière les silences et les non dits autour des besoins de manger et de dormir, l'ampleur de la pauvreté, de la précarité, du vide et de la peur qui marquent le parcours de plusieurs de ces jeunes.

Aujourd'hui, je dois avouer que je suis tout simplement ravie lorsqu'un bâillement vient attester d'une nuit de sommeil respectable ou encore témoigner de la digestion d'un estomac bien rempli par le repas du midi, chez un jeune qui accepte de réapprendre à avoir faim et à avoir sommeil. Cela peut paraître banal, mais il n'en est rien, puisque cette position nouvelle — un véritable succès thérapeutique selon moi — s'inscrit dans l'abandon d'une certaine forme d'omnipotence que contribuait bien souvent à consolider l'usage de substances psychoactives. Accepter la faim et le besoin de sommeil, cela implique de reconnaître sa condition d'humain avec ce que cela comporte de besoins de dépendance et d'ouverture à la négociation relationnelle; une ouverture aussi à certaines formes d'apprentissage, de même que l'assouplissement de défenses, le renoncement à des mécanismes d'adaptation qui se sont avérés utiles et gratifiants à courte échéance mais très coûteux à plus long terme. Du presque banal dans des conditions normales, la question du manger et du dormir tient de l'essentiel et du primordial dans la clinique de la précarité. Ma propre façon d'occuper la cafétéria, d'y manger ou non, est désormais imprégnée de cette conscience des effets dévastateurs de la précarité, désormais marquée par cette prise de conscience des risques auxquels sont exposés de nombreux jeunes marginaux qui doivent composer avec la faim et le manque de sommeil réparateur: manque d'attention, perte de vigilance, difficulté de concentration, fuite en avant dans la prise de risques, pour n'en nommer que quelques uns.

Je comprends mieux aussi pourquoi plusieurs ne profitent pas des douches mises à leur disposition, à moins qu'une démarche quelconque ne les y incite. Car 
se laver correspond à une forme de désir, lequel peut être éteint ou en attente chez le jeune qui demeure dans ses vêtements sales ou souillés. Se laver c'est accepter d'exposer sa peau et de se rencontrer dans sa nudité; une démarche qui peut s'avérer risquée, lorsque cette peau déjà fragile fait office, avec ses attributs, de carapace, de bouclier ou de pare-excitations pour se préserver des contacts peu sûrs ou tenir l'autre à distance. Lorsque les odeurs de corps non lavés ou des vêtements humides envahissent la salle d'accueil du centre de jour lors d'une journée de pluie ou de neige, il m'arrivera de me sentir étouffée ou repoussée par tant de proximité. Trop de bruits, trop d'odeurs, forcée de respirer à petites doses, et me voilà indisposée à manger à la cafétéria et incapable même... de penser ! Dès lors, je peux mieux comprendre pourquoi des jeunes n'arrivent pas à dormir et se sentent envahis dans les refuges qu'ils fréquentent faute de mieux. Incapables de se réconforter ou de se réassurer eux-mêmes, ils sont à risque d'exposer leur vulnérabilité.

Perturbations du Moi, perturbation des fonctions adaptatives, mésadaptation ou mode d'adaptation paradoxale, limites d'un système à intégrer les plus vulnérables? L'évaluation de la situation, étendue sur des jours, des semaines sinon des mois, pourra permettre de mettre à jour certains indices et d'esquisser des pistes. Pendant ce temps, je continue d'interroger un état de vigilance alerte ou le silence d'un corps qui ne produit pas les sons ou les mouvements auxquels on devrait normalement s'attendre.

\section{Le corps des jeunes}

Il est difficile de ne pas être bouleversée par des jeunes dont le corps est abîmé par les gestes répétés de l'injection de drogues: les membres enflés, bleuis ou rougis, troués ou gravés de plaies mal cicatrisées contrastent avec les visages où les expressions de candeur sont à peine camouflées par les marques de négligence ou de violence. Jeunesse avortée, amaigrie, précipitée dans des prises de risques aléatoires et ordaliques... Le corps du jeune toxicomane est un corps chargé, lourd de marques, de traces et de creux, prêt à échouer quelque part lorsqu'il est sous influence ou exténué. C'est un corps qui, lorsque hors de lui-même, peut transmettre la peur et susciter l'effroi. En état altéré de la conscience, c'est un corps qui trouble et qui ne laisse jamais indifférent, synonyme de débordement, d'excès et d'abandon: de la vigilance, de la conscience, de soi.

Le corps des jeunes, tantôt lieu de repères, avec ses tatouages ou autres marques identitaires chargées de me distinguer des autres ou de $m$ 'affilier, tantôt territoire d'appartenance (Aubin, 2000), tantôt chantier à déserter, dévasté par trop de vide qui fait rage à l'intérieur et n'accorde aucun répit. De last resort ou dernier refuge, il peut devenir un corps en orbite, véritable électron libre faisant corps avec la substance psychotrope, ce puissant carburant qui le propulse hors de l'orbite relationnelle dans l'illusion de la toute-puissance toxicomaniaque. Un corps qui fait des sauts périlleux, souvent sans filet de sécurité, tenté de s'affranchir de toute forme d'attachement mais qui finit, le plus souvent, échoué sur sa propre détresse, 
sans mot pour la décrire ou la nommer. Métaphore que tout cela ? C'est qu'il en faut bien pour décrire une réalité d'être insupportable !

Mon propre corps est donc aux aguets, rassuré certes par la présence de mes collègues et l'étayage que le groupe autorise. Mais je me dis parfois que je n'aimerais pas rencontrer sur mon chemin ces corps insensés, ceux de ces jeunes qui nous désarçonnent en nous filant entre les doigts pourrait-on dire, tels des satellites propulsés par une impulsivité spectaculaire et désolante de destructivité. Mais, malgré les dérives toxicomaniaques qui emportent ces jeunes, le dispositif mis en place et la qualité de l'accueil permettent de faire surgir, presqu'à chaque visite, quelque chose de leur vulnérabilité et de leur sensibilité. Certains d'entre eux découvrent, avec nous, qu'ils peuvent être attachants malgré leurs rebuffades, leurs refus et les manifestations de leur révolte ou de leur destructivité.

\section{Le regard et la poignée de main}

Des échanges dans mon bureau se jumellent aux interactions aléatoires dans le centre de jour, régulièrement ponctués de poignées de mains en guise d'ouverture ou de clôture - simple geste de reconnaissance mutuelle qui réintroduit et stimule un certain plaisir de communiquer ou d'être ensemble. La poignée de main est loin d'être anodine puisqu'elle permet, outre l'expression d'un signe de reconnaissance et de respect, d'orienter l'interaction de manière à ce que les regards se croisent, tout en restaurant un espace entre nos deux corps qui autorise la liberté de mouvement et annule toute ambiguïté.

Pour certains jeunes, il est clair que ce contact physique — la plupart du temps initié par moi dans un premier temps, a contribué à la reconstruction de leur espace vital soumis à trop d'intrusion. Ce simple geste me permettait de leur indiquer les limites à ne pas franchir lorsqu'ils pouvaient être tentés de se rapprocher trop près, leur corps vacillant presque dans l'attente d'un geste de ma part pour l'orienter. De plus, pour des jeunes qui ont été captifs de relations abusives ou ambiguës, le contact de nos mains tendues instaure la distance qui leur permet d'oser une plongée moins menaçante dans le regard de l'autre. Là encore, cette avancée n'est pas banale, quand on sait l'importance qu'elle a au plan relationnel, attestant de l'appropriation de son pouvoir sur les choses et d'une ouverture à communiquer.

Le contact physique que constitue la poignée de main me renseignera sur le tonus musculaire et de surcroît sur le moral des jeunes: fatigue extrême ou abandon de soi dans une main qui se dépose mollement dans la mienne, accompagné d'un regard incertain ou fuyant, ou encore crispation méfiante ou farouche d'une autre au contact de la mienne. La poignée de main nous fournira aussi, aux jeunes et à moi-même, un repère stable sur lequel s'appuyer quand l'incertitude et la précarité pèsent trop lourd et que les progrès se font attendre. Elle symbolisera l'accord qui nous permettra de continuer de croire que quelque chose d'un possible puisse advenir; elle permettra de résister au poids du désespoir. Rituel sorti de sa conformité, garant de l'espoir quand la désespérance guette, nourrie de pauvreté et de honte. Je n'aurais jamais cru que ce geste si coutumier 
dans notre société, autrement banalisé dans d'autres contextes, retrouverait autant de signification symbolique. Sa portée thérapeutique est loin d'être négligeable, à la condition bien sûr d'y être attentif, d'en mesurer l'utilisation et d'en évaluer la portée.

Faute d'être sujet de leur propre vie, croulant sous l'impuissance et les échecs répétitifs, se débattant avec une faible estime d'eux-mêmes, la honte, le doute ou la culpabilité, les jeunes que je rencontre ont souvent opté pour des solutions de compromis coûteuses, cherchant à acquérir une valeur et à rehausser leur estime de soi en acceptant de se positionner en objet de désir pour l'autre, se rendant souvent captifs de relations ambiguës ou mercantiles. Ainsi, la prostitution permettra à certains de faire de l'effet, d'impressionner, de séduire, de contrôler momentanément et bien sûr de gagner de l'argent; les activités délinquantes fourniront des récompenses immédiates et parfois des punitions non désirées, mais celles-ci contribueront à stimuler le sentiment d'exister même au péril de sa propre sécurité ou de sa vie. Car rien ne semble pire pour certains que le vide intérieur qui prend au ventre dans les moments de solitude; celle-ci étant d'ailleurs insupportable pour plusieurs.

Les entretiens avec ces jeunes sont certes très exigeants, sollicitant simultanément plusieurs niveaux d'écoute. J'ai souvent l'impression d'être en position d'évaluer leur degré de vitalité, à l'affût de leurs signes vitaux et de ma propre respiration parfois, laquelle peut être tentée de ralentir de manière importante à l'écoute de certaines histoires ou en observant un silence plus proche du mutisme que de la réflexion active. Il m'est souvent arrivé d'ailleurs, en position d'écoute, de placer ma main de manière à palper ma carotide, comme si je cherchais une forme de réassurance ou de repères, là où ils se font vagues et flous dans le discours de jeunes qui consomment surtout des opiacés, ou pour qui la réflexion est court-circuitée par le mode de fonctionnement opératoire dans lequel les maintiennent les psychotropes. À l'écoute de récits entourant certaines prises de risques, je serai prise d'assaut par des états de sidération, d'indignation, d'arrêt de la pensée, de suspension du jugement. Le temps de prendre conscience du processus vicariant, de prendre du recul, de me repositionner, tout en maintenant ma position d'écoute bienveillante. Le récit des abus, des assauts ou des viols dont certains sont victimes, dans le cadre de leurs activités délinquantes ou de prostitution, de même que celui relatant les conséquences de leurs propres accès de rage ou de folie sont certainement les plus difficiles à entendre, à digérer ou métaboliser. La rencontre avec ces funambules n'est jamais banale et comporte des risques que l'on ne doit jamais négliger, sous peine d'entretenir un discours ponctué de bonne volonté mais tout aussi stigmatisant que les préjugés à leur égard.

Ainsi, les face-à-face répétés avec des personnes extrêmement vulnérables, limitées dans leurs capacités d'adaptation à notre société, suicidaires ou tentées par l'inscription dans le négatif, confrontées à leur héritage empreint d'abus ou de violence, $\mathrm{m}$ 'ont amenée à prendre position de manière probablement plus explicite sur les questions de vie, de mort et de prises de risques. Il m'apparaît maintenant 
plus que jamais improbable que mon corps de psychothérapeute puisse exister sans qu'une position éthique détermine le socle sur lequel il va s'appuyer. Accompagner quelqu'un dans la recherche de soi pour une meilleure compréhension, une amélioration de sa situation et même une guérison, constitue certainement une façon de proposer, qu'on le veuille ou non, une manière particulière d'être ou de concevoir l'être humain. J'ajouterais que ma pratique auprès de jeunes en très grande difficulté a certainement contribué à forger la personne que je suis maintenant et orienté mon engagement social.

\section{Le corps de l'intervenant}

Devant les pratiques à risque, les élans de destruction, les tendances et conduites «parasuicidaires» (Shneidman, 1996) ou les dérives toxicomaniaques de plusieurs jeunes que je suis amenée à rencontrer, il y a tous les jours urgence de demeurer vigilant et en contact avec sa propre vitalité, sous peine d'être contaminé par le désespoir, la détresse, le sentiment d'impasse ou encore de subir affres du clivage et de la toute-puissance toxicomaniaque. En effet, le contact avec ces jeunes qui font corps avec la substance, propulsés comme des électrons libres sous l'effet de stimulants ou encore englués par l'absorption d'opiacés n'est pas inoffensif. La nécessité d'élaboration et du prendre soin de soi deviennent ici vitaux, pour contrer le danger potentiel et réel de voir miner sa propre vitalité, soit par la congestion des voies somatiques ou par tout autre manifestation de «traumatisation vicariante» (Perreault, 2006).

Car il s'agit d'un travail qui prend au corps. Les réactions contretransférentielles (non limitées aux interactions dans mon bureau) sont multiples. Elles interpellent brusquement ou sournoisement le corps avant de se traduire en émotions ou affects; elles empruntent la voie de l'inconscient pour réapparaître dans les rêves ou privilégier la voie somatique. Les attaques à l'activité réflexive ne sont pas rares et les coups au ventre fréquents, en termes de revirement d'estomac (estomac qui fait un tour) ou de barres dans le ventre. Certains intervenants se plaignent de maux de tête ou de tensions musculaires, ou encore d'élans soudains d'hyper motricité sans but. Heureusement, l'étayage relationnel va permettre de réduire la portée somatique, par les espaces d'élaboration qu'il autorise: échanges avec des collègues, réunions d'équipe, rencontres de supervision ou de consultation clinique, démarche de psychothérapie. J'ai personnellement recours depuis plusieurs années déjà à une forme de mise en forme du corps — héritage de mon parcours dans le domaine des arts dramatiques, qui me permet de renforcer ma résistance au stress physique et psychologique tout en me gardant alerte et souple sur le plan émotionnel. Toutefois, pour être efficace, cette activité se doit d'être régulière et suffisamment investie. Toute activité qui permet d'assouplir ou de détendre les muscles internes (le diaphragme est particulièrement sollicité) et de favoriser la respiration profonde s'avère aussi particulièrement pertinente: exercices de respiration, chant, jeu dramatique, danse, yoga, parmi d'autres. 
Loin de moi l'idée de généraliser ces symptômes à tout intervenant qui va à la rencontre de ces jeunes, mais la nature des mécanismes d'adaptation et de défense mis en branle chez ceux-ci, dont participent très souvent la dissociation des affects et le clivage du Moi, suscitent sans conteste des réactions importantes chez l'intervenant ou le psy qui s'efforce de préserver l'activité de penser. Des jeunes qui ont bénéficié d'un soutien psychothérapeutique et augmenté tant soit peu leur vigilance et la conscience de leurs limites se sont permis de commenter mon attitude «calme» et «posée » et m'ont fait remarquer que je respirais «par le nez» (leurs mots entre guillemets)! Des activités de jeu dramatique proposées aux jeunes m'ont permis d'observer la difficulté que plusieurs éprouvent à respirer avec amplitude ou de manière régulière ou contrôlée (inspirer, retenir, expirer). Leur attitude paraît souvent méfiante face à cet exercice pourtant si simple, en apparence.

\section{Le risque d'enfermement}

Ma pratique auprès de jeunes en grande difficulté et des intervenants qui les accompagnent m'amène à composer avec l'inconfort, tissé d'anxiété, de crainte, d'inquiétude ou de révolte, par rapport à ce que ces jeunes s'infligent eux-mêmes mais aussi par rapport à la mauvaise foi ou certains travers de notre société. Les jeunes que je rencontre ne sont pas seulement victimes ou à risque d'abandon, ils sont aussi à risque de disqualification — que celle-ci vienne d'eux-mêmes ou de la société.

Quand ils ne sont pas atteints du «syndrome d'auto-exclusion» (Furtos, 1994), plusieurs courent le risque d'être stigmatisés par le court-circuitage presque systématique de l'évaluation de leur situation et il n'est pas rare de constater la réduction de leurs problèmes à une entité diagnostique. Il m'est régulièrement donné d'observer que la collaboration de proches ou de partenaires n'est pas prise en compte dans le plan d'intervention. Elle est souvent évacuée, au pire discréditée, alors qu'elle pourrait selon mon expérience contribuer à sortir de l'impasse, à générer de l'espoir, ouvrir sur des avenues créatrices et même générer des solutions auxquelles nous n'avions osé penser. Je m'insurge donc régulièrement contre les préjugés qui accablent ces jeunes, que ceux-là viennent d'eux-mêmes ou d'ailleurs, régulièrement indignée de l'injustice qu'ils véhiculent ou de la mauvaise foi qui les accompagne.

Bien sûr, les comportements de plusieurs jeunes qui passent par la rue ou qui prennent des risques hasardeux présentent des similitudes avec divers troubles psychiatriques. Mais j’y vois aussi la mise en scène mal orchestrée de besoins psychologiques non comblés qui demandent à être vus, entendus ou validés de toute urgence, pourrait-on dire. Là où on peut lire une faiblesse du Moi ou voir les conséquences de prises de risques coûteuses, il peut y avoir aussi une sensibilité particulièrement aiguisée à l'environnement ou aux besoins psychologiques des figures d'attachement. Le corps du jeune de la rue, tenté par l'ancrage dans l'errance, l'isolement ou l'addiction, est parfois un corps sacrifié ou qui se 
sacrifie ; tantôt bouc-émissaire, tantôt corps sous tension, histrionique, annonciateur de drames inconscients, révélateur de souvenirs traumatiques. Un corps qui, somme toute, s'oppose au refoulement et donc à une certaine normalité. Comme certains personnages d'Anne Hébert, c'est «le corps tout entier qui exprime les signes d'émotions » (Brulotte, 1997, 155). Un corps qui demande à être décrypté, un corps qui parle pour soi mais duquel peine à émerger un Moi différencié des fantômes qui l'habitent et qui brouillent sa conscience.

C'est pourquoi j'accorde une grande importance à l'exploration de l'histoire personnelle et familiale des jeunes, source d'indices et d'informations précieuses: secrets de famille, événements ou détails permettant de faire la lumière et de résoudre l'impasse. La complexité des messages que nous envoient les jeunes en grande difficulté appelle à la prudence, à l'échange, à la vigilance et à la rigueur. Ces jeunes sont à risque de toutes sortes d'enfermement et je crois que nous sommes à risque d'être fortement tentés par le pouvoir de savoir à leur place, tellement les situations dans lesquelles ils baignent sont sources d'anxiété. Sans faire fi de l'avancement des connaissances et de ce que celles-ci ont permis de produire en termes de traitements et de services, je pense que nous sommes très à risque de verser dans le confort d'une certitude alimentée de préjugés, en ce qui a trait à notre approche des jeunes en difficulté. Les outils et les connaissances dont nous disposons pour mieux comprendre ces problématiques ne devraient pas servir à réduire notre compréhension dans un cadre privé de la prise en compte du contexte et de l'histoire de chacun. Aussi, le passage de vouloir aider et soigner à contraindre peut se faire furtivement sous le signe de la bonne foi, alors que ces jeunes nous invitent, comme le font d'autres jeunes au parcours plus normatif, à jongler avec nos certitudes afin de leur laisser un peu de place pour créer ce qu'ils adviendront. Je crois sincèrement qu'une société s'enrichit toujours à l'écoute de ses populations vulnérables, à la condition que cette forme d'écoute puisse permettre de mettre en perspective les bons coups et les moins bons coups d'une collectivité, en s'intéressant aux facteurs qui contribuent à éloigner certains parcours de la norme.

Je suis confrontée régulièrement, avec mes collègues intervenants, à des impasses qui sollicitent tous les jours notre créativité, en plus d'être témoin avec eux de situations qui rentrent dans le corps comme on le dit chez nous d'une épreuve. Par ailleurs, refuser de prendre en compte les multiples avenues que le corps peut emprunter pour parler, se taire, interpeller ou fuir, ou refuser de se laisser interpeller par les attributs qui parent ou marquent le corps adolescent ou jeune adulte, c'est risquer de court-circuiter l'échange, au pire, de rompre toute communication entre ces jeunes et nous. C'est risquer de réduire la complexité des messages qu'ils portent, de les enfermer dans des étiquettes diagnostiques qui orienteront, le plus souvent négativement, le regard d'autrui à leur égard. Affirmer, par exemple, qu'ils sont (sic) un trouble de personnalité ne résout souvent que peu de choses, surtout si on n'a rien à leur offrir comme possibilité de soins, comme c'est souvent le cas pour les jeunes que je rencontre puisque les caractéristiques de leur problématique ne rencontrent pas très souvent les critères de sélection des 
services offerts dans la communauté. Ils ont trop de ceci, pas assez de cela, et surtout, ils demandent qu'on leur accorde du temps. Il m'arrive donc assez fréquemment d'éprouver une profonde indignation par rapport aux préjugés à leur égard, lesquels les menacent d'enfermement dans une classe sociale à part, voire négligeable. Sans que nous nous en rendions compte, ces jeunes reçoivent souvent le mandat de réussir à enclencher leur processus de résilience le plus vite possible, sous peine de se voir réduire leur temps de reprise ou retirer leur droit à l'échec. Dans une société friande de héros, de performance et de rendement, le temps à accorder aux personnes défavorisées ou les rejects comme disent les jeunes, semble diminuer... sauf quand ceux-ci parviennent à nous toucher ou nous séduire, par leur performance à devenir des self-made men ou women!

Ma pratique dans un centre de jour qui accueille ces jeunes décrocheurs, en échec ou à risque de toutes sortes m'a amenée, avec mes collègues et partenaires, à proposer des avenues trop peu explorées à ce jour dans nos structures de soins, de celles qui interpellent directement le corps de ces jeunes : des activités diverses, sportives, artistiques ou de cirque leurs sont proposées lors de leurs passages au centre. Ces modalités d'intervention, alliées à «l'observation phénoménologique » (Bergeret et Dubor, 1972, 2000, 133) contribuent à la reconnaissance d'un désir d'être en relation, sans qu'une demande d'aide ne soit exigée comme pré-requis à l'activité. En ce qui concerne mes rencontres avec eux, je proposerai à point donné des modalités d'expression telles que le dessin, le jeu de rôle ou des mises en situation qui vont permettre de dénouer des impasses en réactivant le travail de l'imaginaire. Je m'intéresserai au corps des jeunes en interrogeant ceux-ci par rapport aux attributs dont ils se parent et en m'attardant au «langage cutané » (Garel, 2008, 229) : habillement, tatouages, piercings, accessoires. Je leur proposerai des exercices ayant pour objectif la prise de conscience du fonctionnement de leur respiration et des points d'appui des émotions dans leur corps. Les démarches qui permettent d'interroger le «vécu somatique (Bolsanello, 2005, 96) méritent certainement d'être explorées là où les solutions addictives peuvent conduire à l'élaboration de véritables «théâtres somatiques » (McDougall, 1989, 15). Interroger le corps, ce qu'il veut dire et exprimer, mais aussi ce qu'il tente de retenir, de garder sous silence...

\section{Conclusion}

La rencontre des jeunes de la rue ou en grande difficulté est souvent bouleversante et peut engendrer des états très divers chez l'intervenant. Il est vrai que le corps en prend souvent un coup, encaissant des états parfois diamétralement opposés, pouvant aller de la sidération à l'émerveillement. Une écoute faite d'empathie et d'authenticité, associée à un contexte d'intervention pluridisciplinaire et une approche ancrée dans la réflexion peut permettre à l'intervenant de demeurer créatif et de ne pas rester subjugué ou captif d'émotions et d'affects très intenses.

La plupart des difficultés auxquelles nous sommes confrontés, avec ces jeunes qui utilisent nos services, suscitent dans un premier temps des sensations 
d'écrasement ainsi que des sentiments de découragement ou d'indignation. L'étayage relationnel que permet le travail d'équipe ainsi que les années d'expérience permettent d'atténuer ces sensations sans toutefois les estomper complètement, ce qui est bon signe évidemment. Il serait malsain de s'habituer à la misère et à la détresse avec les risques que cela pourrait comporter de la minimiser ou de la banaliser, au pire, de s'y attacher et de s'en nourrir! La rencontre et l'accompagnement de jeunes en difficulté représentent donc une confrontation avec ses propres limites, mais aussi une invitation à les dépasser. Dit autrement, côtoyer la précarité, la pauvreté et la destructivité appelle à la créativité : inventer, imaginer, au risque d'être dépassé par les événements et de crouler sous l'impact vicariant de la souffrance observée et déposée en nous. Outre mes propres valeurs et les repères qui vont influencer l'orientation que prendra mon écoute, mon corps physique avec sa peau, sa respiration, ses sens (odorat, vue, ouïe, toucher) et ses organes internes - va entrer en résonance avec celui des jeunes et servir tantôt de réceptacle ou de contenant, tantôt de baromètre, tantôt de guide, pour tenter de ne pas nuire d'abord, comprendre ensuite et orienter de manière créatrice, autant que faire se peut, des parcours marqués par la destructivité, l'échec ou le mortifère.

Mon expérience dans un centre de jour qui accueille des jeunes dits sans abri, de la rue ou en rupture, m'a amenée à opter pour une approche foncièrement humanitaire, alliant les caractéristiques de la psychologie communautaire aux regards ethnologique et phénoménologique. La prise en compte de l'interaction s'avère tout aussi essentielle afin de comprendre le parcours de ces jeunes et les soutenir dans le «réaménagement de (leur) dépendance» (Jeammet et Corcos, 2001) et la reconstitution de leur Moi-peau vers un Moi-pensant (Anzieu, 1994).

La rencontre avec les jeunes en grande difficulté constitue certainement un face-à-face avec ses propres limites, celles des institutions et celles de notre société. Elle interpelle tous les jours notre conscience morale et notre créativité, dans la mesure ou se satisfaire des limites équivaudrait souvent à renoncer, démissionner, condamner ou abandonner. Là où la déshumanisation mine les potentialités humaines, nous sommes conviés à générer de l'humanité; les diverses missions humanitaires en font foi. Tisser de l'humanité en produisant des rencontres avec l'altérité, en proposant et investissant des lieux de subjectivation, pour contrer les diverses formes de déliaison, de dissociation ou de fuite dans la toute-puissance. Utopie que de penser que la solidarité puisse venir à bout d'un courant social favorisant la construction de self-made men? Il s'agit très certainement d'emprunter une position clinique éloignée de l'isolement et de l'omnipotence, pour rejoindre des jeunes à risque d'y demeurer.

diane aubin

1664 , rue ontario est montréal (québec) h2l 1s7

d_aubin@videotron.ca 


\section{Note}

1. Le centre de jour: cafétéria; école; infirmerie; service à la famille; service de consultation et d'écoute psychothérapeutique; ateliers d'art, de musique et d'informatique; distressing room; programmes d'insertion et d'intégration au travail ; mentorat; activités en partenariat; sorties culturelles et sportives; séjours de rupture; etc. www.danslarue.org

\section{Références.}

Anzieu, D., 1994, Le Penser, Du Moi-peau au Moi-pensant, Paris, Dunod.

Aubin, D., 2008, Les jeunes en difficulté, Une invitation à la créativité, Psychologie Québec, 25 (02), 24-27.

Aubin, D., 2002, Rejoindre les jeunes de la rue, PRISME, nº 37, 144-150.

Aubin, D., 2000, Le corps, lieu de repères pour les jeunes de la rue ou la quête d'un territoire d'appartenance, Santé mentale au Québec, XXV (2), 90-108.

Bergeret, J., Dubor, P., 1972, 2000, Entretien avec le patient en psychologie pathologique, in Jean Bergeret et collaborateurs, Psychologie pathologique, théorique et clinique, Paris, Masson, 133-139.

Birraux, A., 2004, Le corps adolescent, Paris, Bayard.

Bolsanello, D., 2005, La pertinence de l'éducation somatique auprès des toxicomanes: le corps vécu, Psychotropes, $11(1), 91-107$.

Bruckner, P., 2000, L'euphorie perpétuelle, Paris, Grasset.

Brulotte, G., 1997, La représentation du corps chez Anne Hébert, in Actes du colloque de la Sorbonne, Anne Hébert, parcours d'une æuvre, Montréal, Éditions de l'Hexagone, 149-161.

Cyrulnik, B., 2001, Drogue et attachement, in Claude Olievenstein, Toxicomanie et devenir de l'humanité, Paris, Odile Jacob.

Flamand, S. et Aubin, D., 2004, «On m'abandonne, je m'abandonne... ?», L'expérience d'un centre de jour comme générateur de changement pour les jeunes de la rue, PRISME, n 44, 136-150.

Fœlsch, P. A., Odom, A. E., Kernberg, O. F., 2008, Modifications à la psychothérapie focalisée sur le transfert (PFT) pour le traitement des adolescentes avec une identité diffuse, Santé mentale au Québec, XXXIII, 1, 37-60.

Furtos, J., 1994, Souffrir sans disparaître (pour définir la santé mentale au-delà de la psychiatrie), in Jean Furtos et coll., Déqualification sociale et psychopathologie, les désirs et limites de la psychiatrie publique, Lyon-Bron, ORSPERE-ONSMP, 9-38.

Garel, P., 2008, L'automutilation superficielle à l'adolescence: le corps dans tous ses états, Cahiers critiques de thérapie familiale et de pratiques de réseaux, Paris, De Boeck Université, n 40, 227-235.

Gotman, A., 2001, Le sens de l'hospitalité, essai sur les fondements sociaux de l'accueil de l'autre, Paris, P.U.F.

Hachet, P., 2005, La toxicomanie : du corps troué aux intrusions psychiques, Imaginaire \& Inconscient, $\mathrm{n}^{\circ}$ 16, 67-75.

Hachet, P., 2001, Ces ados qui jouent les kamikazes, Paris, Éditions Fleurus.

Jacobson, E., 1954, Transference problems in the psychoanalytic treatment of severely depressive patients, Journal of the American Psychoanalytic Association, 2, 595-606.

Jeammet, P. et Corcos, M., 2001, Évolution des problématiques à l'adolescence, L'émergence de la dépendance et ses aménagements, Paris, Doin éditeurs.

Lambrette, G, 2008, À son corps défendant, Une approche constructiviste du corps dans le champ des toxicomanies, Psychotropes, 14, 9-21.

Le Breton, D., 2007, En souffrance, Adolescence et entrée dans la vie, Paris, Éditions Métailié.

Le Breton, D., 2002, Conduites à risque, Paris, P.U.F.

Le Breton, D., 1990, Anthropologie du corps et modernité, Paris, P.U.F. 
Filigrane, automne 2009

McDougall, J., 1989, Théâtres du corps, Paris, Éditions Gallimard.

Otero, M. , 2006, La santé mentale enfermée dans le corps et la norme, Équilibre, 1 (1), 33-47.

Perreault, C., 2006, Perturbation et fatigue... Croissance et enthousisame..., Équilibre, 1 (2), 45-54.

Pirlot, G., 2002, Complexité psychopathologique du phénomène d'addiction réévalué avec des concepts psychosomatiques et métapsychologiques, Psychotropes, 8 (2), 97-118.

Pommereau, X., 2006, Ado à fleur de peau, Paris, Éditions Albin Michel.

Ratier, F., 2007, Abord clinique de la grande précarité, EMPAN, n 66, 40-48.

Shneidman, E. S., 1996, Le tempérament suicidaire, Risques, souffrances et thérapies, Paris, De Boeck \& Belin, 1999. 